\title{
Development and Characterization of a Hydrogel Containing Silver Sulfadiazine for Antimicrobial Topical Applications
}

\author{
KARIN S. P. JODAR, ${ }^{1}$ VICTOR M. BALCÃO, ${ }^{1,2,3}$ MARCO V. CHAUD, ${ }^{1}$ MATTHIEU TUBINO, ${ }^{3}$ VALQUÍRIA M. H. YOSHIDA, \\ JOSÉ M. OLIVEIRA JR, ${ }^{1}$ MARTA M. D. C. VILA ${ }^{1,3}$ \\ ${ }^{1}$ LaBNUS - Biomaterials and Nanotechnology Laboratory, i(bs) ${ }^{2}$ - Intelligent Biosensing and Biomolecule Stabilization Research Group, \\ University of Sorocaba, Sorocaba, São Paulo, Brazil \\ ${ }^{2} \mathrm{CEB}$ - Centre of Biological Engineering, University of Minho, Braga, Portugal \\ ${ }^{3}$ Institute of Chemistry, University of Campinas, Campinas, São Paulo, Brazil
}

Received 26 January 2015; revised 8 April 2015; accepted 13 April 2015

Published online 7 May 2015 in Wiley Online Library (wileyonlinelibrary.com). DOI 10.1002/jps.24475

\begin{abstract}
Development and optimization of a hydrogel with impregnated silver sulfadiazine was pursued, for antimicrobial topical applications. The selected hydrogel exhibited a homogeneous appearance, with whitish colloration and devoid of any fractures or cracks. The content in impregnated silver sulfadiazine was within established limits $(1 \%$, w/w) with a standard deviation of up to $1.28 \%$. The hydrogel presented a good characteristic in relation to release of the active antimicrobial principle, verified through swelling tests and antimicrobial activity. The swelling tests indicated a higher increase in weight during the first $6 \mathrm{~h}$ of contact with a moist environment, with a maximum value of $266.00 \pm 0.81$, and with maintenance of the original shape of the hydrogel. The impregnated silver sulfadiazine presented antimicrobial activity, as expected, indicating a prolonged release of the drug. The infrared spectra of the hydrogel with impregnated silver sulfadiazine indicated that the drug did not engage in any bonds with the polymeric matrix, which otherwise could have reduced its antimicrobial activity. The mechanical resistance tests produced good results, indicating that the hydrogels may be utilized in different locations of the human body with skin lesions. (c) 2015 Wiley Periodicals, Inc. and the American Pharmacists Association J Pharm Sci 104:2241-2254, 2015
\end{abstract}

Keywords: hydrogels; PVA; silver sulfadiazine; antimicrobial topical applications; physical characterization; polymeric drug delivery systems; skin; transdermal drug delivery

\section{INTRODUCTION}

Among the various dosage forms developed as modified-release systems, hydrogels can be considered controlled-release systems for drugs of topical application, depending on both the type of formulation and application in which they are used. ${ }^{1}$ Hydrogels are three-dimensional (3D) networks of hydrophilic polymers that, in contact with water, swell and may release the drug by different mechanisms. ${ }^{2}$ In this sense, hydrogels are of particular interest in the treatment of topical wounds because of their intrinsic low toxicity, potential for extended release of drugs, and the ability to keep the wound hydrated. ${ }^{3-5}$ Review studies have pointed toward the use of various types of natural and/or synthetic polymers for the preparation of hydrogels, such as poly(acrylic acid), poly(ethylene oxide), poly(ethylene glycol) (PEG), poly(vinyl pyrrolidone), polyvinyl alcohol (PVA), polyglycerol esters of fatty acids, carbomers, sodium alginate, chondroitin sulfate, pectin, dextran, carboxymethylcellulose, and other cellulose derivatives, chitosan, gelatin, and gums. ${ }^{6-11}$ PVA is extensively used to produce hydrogels aiming at the treatment of topical ulcers. PVA is a nontoxic, biocompatible polymer that has excellent film-forming properties and ease of processability, as well as mechanical, thermal, and chemical resistances. ${ }^{5,10,12,13}$ Dextrans are also widely used for biomed-

Correspondence to: Marta M. D. C. Vila (Telephone: +55-15-2101-7181 Fax: +55-15-2101-7000; E-mail: marta.vila@prof.uniso.br)

Journal of Pharmaceutical Sciences, Vol. 104, 2241-2254 (2015)

(C) 2015 Wiley Periodicals, Inc. and the American Pharmacists Association ical applications because of their biocompatibility and ease of modification and biodegradation. ${ }^{14}$ For the treatment of skin lesions, among various substances, there is a consensus in the use of silver sulfadiazine at $1 \%(\mathrm{w} / \mathrm{w})$ for the treatment of burns and skin infections. Silver sulfadiazine is effective against a wide microbiota of gram-negative bacteria such as Escherichia coli, Enterobacter, Klebsiella, and Pseudomonas aeruginosa, including also gram-positive bacteria such as Staphylococcus aureus and yeasts such as Candida albicans. ${ }^{15}$ Silver sulfadiazine is indicated in the treatment and prevention of infections in burns, with classification of anti-infectant agent in the form of cream at $1 \%(\mathrm{w} / \mathrm{w})$; short-term adjuvant treatment, for infections in both leg ulcers and pressure ulcers; prophylaxis of infection in areas of skin grafts prone to abrasion. ${ }^{16}$ Silver sulfadiazine in the form of cream requires multiple daily applications, interfering with the healing process as the wound dressing exposes patients to infectious agents, in addition to the pain and trauma that it causes because the cream is not biodegradable and requires removal before reapplication. ${ }^{17}$ Considering the growing importance of the pharmaceutical form hydrogel, the aim of the present research work was to develop and evaluate a hydrogel using PVA and/or dextran as raw materials, containing incorporated silver sulfadiazine, for antimicrobial topical applications. The optimized hydrogel formulation integrating silver sulfadiazine was subsequently fully characterized physicochemically, encompassing determination of pore size and porosity via X-ray tomography, surface morphology via scanning electron microscopy (SEM), thermal analyses via thermogravimetric analysis (TGA), and differential scanning 
calorimetry (DSC), Fourier transform infrared (FTIR) spectrophotometry, and X-ray diffraction (XRD) analyses.

\section{MATERIALS AND METHODS}

\section{Materials}

\section{Chemicals}

All reagents used were of analytical grade or better, and were used without further purification. Tap water was purified in a Milli-Q Elga Purelab system (Molsheim, France) to a final conductivity of approximately $18.2 \mathrm{M} \Omega \mathrm{cm}^{-1}$. PVA with a hydrolysis degree of $89 \%$ (PVA89), aqueous glutaraldehyde solution $(25 \%, v / v)$, and PEG 4000 were purchased from Dinâmica Produtos Químicos Ltda (Diadema, São Paulo, Brazil). PVA with a hydrolysis degree of $98.99 \%$ (PVA99) was purchased from Sigma-Aldrich (St. Louis, Missouri). Dextran from Amresco Biochemicals Life Research Products was acquired from Sellex (São Paulo, Brazil). Silver sulfadiazine with a purity degree of $100.06 \%$, considering $29.3 \%$ silver, was purchased from Valdequímica Produtos Químicos Ltda (São Paulo, Brazil). The ointment with silver sulfadiazine at $1 \%(\mathrm{w} / \mathrm{w})$, with tradename Dermazine ${ }^{\circledR}$ and manufactured by Silvestre Labs Química Farmacêutica Ltda (Rio de Janeiro, Brazil) was purchased from the local market at the city of Sorocaba (Sorocaba, São Paulo, Brazil).

\section{Biological Materials}

The bacterial strain utilized in the antimicrobial assays was Staphylococcus aureus CCCD S007, and was acquired from Cefar Diagnóstico Ltda (São Paulo, Brazil). The microbiological growth media utilized were BHI broth (Brain Heart Infusion) from Fluka Analytical (St. Louis, Missouri) and Manitol Salt Agar from Prodimol Biotecnologia S.A. (São Paulo, Brazil).

\section{Analytical Equipment}

Fourier transform infrared spectra were gathered in a FTIR Spectrophotometer from Agilent (model Cary 630; Santa Clara, California). X-ray diffractograms were gathered in a X-ray diffractometer from Shimadzu (model XRD7000; Kyoto, Japan). Thermogravimetric characterization of the hydrogels was accomplished via thermogravimetric analysis (TGA), whereas thermal analyses were pursued by DSC. TGA analyses were carried out using a thermogravimeter from TA Instruments (model 2050; New Castle, Delaware), whereas the DSC analyses were carried out using a differential scanning microcalorimeter from TA Instruments (model MDSC 2910).
The surface of the hydrogels containing incorporated silver sulfadiazine was duly observed in a scanning electron microscope (model JSM-63660 CV; JEOL, Tokyo, Japan). Hydrogel samples were sputter-coated with colloidal gold under vacuum, and placed in the microscope chamber. Microphotographs were gathered using electron beams with acceleration speeds of $10-20 \mathrm{keV}$. The samples were randomly scanned and photomicrographed at magnifications of $\times 50, \times 200$, and $\times 1000$. A computed tomographer via X-ray transmission from Bruker microCT (model SkyScan 1174; Kontich, Belgium), and an energydispersion X-ray fluorescence (EDXRF) spectrometer from Shimadzu (model EDX-700) were utilized in all nondestructive analyses, for gathering tomographic images of the hydrogels. The analysis software utilized for processing the tomographic images were CTVox ${ }^{\text {TM }}$ (version 2.6.0 r908-64bit, from Bruker microCT) and $\mathrm{CTan}^{\mathrm{TM}}$ (version 1.13.5.1-64bit, from Bruker microCT), and CTvol (version 2.2.3.0-64bit, from Bruker microCT).

\section{Experimental Procedures}

\section{Preparation of Hydrogels}

To produce the hydrogels, aqueous dispersions of PVA99 (10\%, $\mathrm{w} / \mathrm{v})$, PVA89 $(10 \%, \mathrm{w} / \mathrm{v})$, dextran $(15 \%, \mathrm{w} / \mathrm{v})$, and silver sulfadiazine $(10 \%, w / v)$ were previously prepared. One-hundred gram PVA (99 or 89) was weighed in an analytical scale, added with $1000 \mathrm{~mL}$ ultrapure water and the resulting mixture kept under magnetic stirring $(\sim 300 \mathrm{rpm})$ and heating $\left(\sim 80 \pm 5^{\circ} \mathrm{C}\right)$ during approximately $2 \mathrm{~h}$, using a magnetic stirring device from Fisaton (model 752 A; São Paulo, Brazil). Following this time period, the mixture was stirred during approximately 6 min at $5200 \mathrm{rpm}$ using an UltraTurrax (model T25D; IKA Werke GmbH and Company KG, Staufen, Germany) homogenizer. Afterwards, the mixture was left under mechanical stirring at $80 \pm 5^{\circ} \mathrm{C}$ for approximately $2 \mathrm{~h}$. The dextran aqueous solution at $15 \%(\mathrm{w} / \mathrm{v})$ was prepared by adding $150 \mathrm{~g}$ dextran with $1000 \mathrm{~mL}$ ultrapure water, with mechanical stirring in a magnetic stirring device $(\sim 300 \mathrm{rpm})$ at room temperature $\left( \pm 25^{\circ} \mathrm{C}\right)$ during approximately $10 \mathrm{~min}$. The aqueous solution of silver sulfadizine at $10 \%(\mathrm{w} / \mathrm{v})$ was prepared by dispersing $10 \mathrm{~g}$ of silver sulfadiazine in $100 \mathrm{~mL}$ of PEG 4000 at room temperature $\left( \pm 25^{\circ} \mathrm{C}\right)$. Eleven hydrogel formulations and two crosslinking methodologies were proposed and duly tested, being one a chemical method (using glutaraldehyde as cross-linking agent) and the other a physical one (cryogelification). All hydrogel formulations tested are described in Table 1. PVA dispersions (either PVA99 or PVA89) and dextran were mixed and heated up to $80 \pm 5^{\circ} \mathrm{C}$ under magnetic stirring $(\sim 300 \mathrm{rpm})$ for

Table 1. Formulations Tested for Hydrogels of PVA/Dextran with Silver Sulfadiazine

\begin{tabular}{|c|c|c|c|c|c|c|c|c|c|c|c|}
\hline \multirow[b]{2}{*}{ Component $(\%, \mathrm{w} / \mathrm{w})$} & \multicolumn{11}{|c|}{ Hydrogel Formulation } \\
\hline & $\mathrm{I}^{a}$ & $\mathrm{II}^{a}$ & $\mathrm{III}^{a}$ & IV & $\mathrm{V}^{a}$ & $\mathrm{VI}^{a}$ & $\mathrm{VII}^{a}$ & $\mathrm{VIII}^{a}$ & IX & $\mathrm{X}$ & $\mathrm{XI}$ \\
\hline PVA99 & 20 & 15 & 10 & 12.5 & 15 & 15 & 5 & - & 10 & 7.5 & 5 \\
\hline PVA89 & - & - & 10 & - & 5 & 5 & 15 & 20 & - & - & - \\
\hline Dextran & - & 4.5 & - & 5.25 & - & 4.5 & 4.5 & - & 6.75 & 8.25 & 9.75 \\
\hline PEG 4000 & 10 & 10 & 10 & 10 & 10 & 10 & 10 & 10 & 10 & 10 & 10 \\
\hline Silver sulfadiazine & 1 & 1 & 1 & 1 & 1 & 1 & 1 & 1 & 1 & 1 & 1 \\
\hline Ultrapure $\mathrm{H}_{2} \mathrm{O}$, ast & 100 & 100 & 100 & 100 & 100 & 100 & 100 & 100 & 100 & 100 & 100 \\
\hline
\end{tabular}

${ }^{a}$ For the chemical cross-linking process, $5.0 \mathrm{~mL}$ of glutaraldehyde aqueous solution at $25 \%$ (v/v) were added.

ast, amount sufficient to; PVA99, polyvinyl alcohol with 99\% degree of hydrolysis; PVA89, polyvinyl alcohol with $89 \%$ degree of hydrolysis. 
approximately $10 \mathrm{~min}$. Subsequently, the silver sulfadiazine solution at $10 \%(\mathrm{w} / \mathrm{w})$ was incorporated and the resulting dispersion was maintained under magnetic stirring for an extra $30 \mathrm{~min}(\sim 300 \mathrm{rpm})$. After this time period, the dispersions were kept at rest until temperature equilibration with room temperature $\left( \pm 25^{\circ} \mathrm{C}\right)$. Subsequently, $5.0 \mathrm{~mL}$ glutaraldehyde solution at $25 \%(\mathrm{v} / \mathrm{v})$ were added, the resulting dispersion duly homogenized, poured into plastic molds, and dried at $60^{\circ} \mathrm{C}$ in a Fanem incubator (model 515 A; Karnataka, India). After completion of the hydrogel's polymerization process, the hydrogels were thoroughly washed with phosphate buffer $\mathrm{pH}$ 7.0, so as to virtually eliminating all nonreacted (free) glutaraldehyde. The hydrogel samples were then cut $\left(2.5 \times 3.0 \mathrm{~cm}^{2}\right)$ wrapped in PVC (polyvinyl chloride) film and stored in the dark. For those hydrogel formulations prepared via criogelification, following homogenization and rest, they were poured into plastic molds and kept at $-4^{\circ} \mathrm{C}$ during $14 \mathrm{~h}$ and then at $25^{\circ} \mathrm{C}$ during $2 \mathrm{~h}$, for five consecutive cycles. The hydrogels were then dried in an incubator set at $60^{\circ} \mathrm{C}$.

\section{Morphological Characteristics}

The hydrogel films formed were initially analyzed with respect to their macroscopic morphological characteristics, considering the absence of air bubbles, the presence of cracks and homogeneity. This evaluation was performed by both naked eye and magnifying glass. Thickness of the hydrogel films was determined with the aid of a manual measuring caliper from Mitutoyo Sul Americana Ltda (Suzano, Brazil). Each hydrogel film was measured at 10 different points, and the average thickness and associated standard deviation were duly calculated.

\section{SEM Analyses}

The surface of the hydrogel films containing silver sulfadiazine was duly observed in a scanning electron microscope (from JEOL; model JSM-63660). Hydrogel samples were coated with colloidal gold under vacuum, and placed in the microscope chamber. Microphotographs were gathered using electron beams with $10 \mathrm{keV}$ energy.

\section{FTIR Spectrophotometry Analyses}

The infrared spectra of samples of silver sulfadiazine, and of samples of selected hydrogels (viz., formulation III) without and with incorporated silver sulfadiazine, were gathered using an FTIR spectrophotometer from Agilent (model Cary 630) in the wavelength range from 4000 to $400 \mathrm{~cm}^{-1}$, with a resolution of $4 \mathrm{~cm}^{-1}$ and using Happ-Genzel apodization.

\section{XRD Analyses}

X-ray diffractograms of silver sulfadiazine, and of samples of selected hydrogels (viz., formulation III) without and with incorporated silver sulfadiazine, were gathered in a X-ray Diffractometer from Shimadzu (model XRD7000), using X-ray radiation from a copper lamp with radiation $\mathrm{K} \alpha(\lambda=1.5418 \AA)$ filtered through a $\mathrm{Cu}$ target. The X-ray scanning was performed at diffraction angles of $2-\theta$ (from $5^{\circ}$ to $90^{\circ}$, with increments of $0.02^{\circ}$ and rate of $2^{\circ} / \mathrm{min}$ ), with a voltage of $40 \mathrm{kV}$, electric current intensity of $30 \mathrm{~mA}$, and X-ray power of $3 \mathrm{~kW}$.

\section{Mechanical Resistance Properties}

Mechanical properties of the hydrogels were evaluated using a texturometer from Stabile Micro Systems (model TA-TX Plus;
Godalming, United Kingdom), evaluating parameters such as resistance to perforation, relaxation, and resilience. The determination parameters were set as distance of $5 \mathrm{~mm}$ for the perforation resistance tests, distance of $2 \mathrm{~mm}$ for both resilience and relaxation tests, and a maximum force of $5 \mathrm{~kg}$ for all tests. All determinations were performed in triplicate.

\section{Thermal Analyses via TGA and DSC}

Thermogravimetric characterization of the hydrogel formulations was accomplished via TGA, whereas thermal analyses were pursued by DSC. The TGA analyses were carried out in a thermogravimeter from TA Instruments (model 2050), using alumina pans for containing the hydrogel samples under inert (argon) atmosphere. For the thermogravimetric analyses, samples were heated from approximately $20^{\circ} \mathrm{C}$ up to $500^{\circ} \mathrm{C}$, and data (weight loss) were gathered at a sampling rate of $2.0 \mathrm{~s}$ per data point. For the DSC analyses, one has used a differential scanning microcalorimeter also from TA Instruments (model MDSC 2910). For every calorimetric assay, approximately 7 (between 6.48 and 7.93) $\mathrm{mg}$ of hydrogel formulation were weighed directly into the interior of high-pressure aluminum pans, and duly sealed by pressure. A reference aluminum pan was also prepared by simply sealing air inside an empty case. The samples were then subjected to a linear temperature increase from approximately $20^{\circ} \mathrm{C}$ up to $250^{\circ} \mathrm{C}$, at a constant heating rate of $10^{\circ} \mathrm{C} / \mathrm{min}$, under an inert atmosphere maintained with a constant flow of argon of $50 \mathrm{~mL} / \mathrm{min}$, during which the amount of heat absorbed by the hydrogel samples was recorded. During the analyses, data (heat absorption) were gathered at a sampling rate of $0.2 \mathrm{~s}$ per data point.

\section{Swelling Degree}

The degree of swelling of the hydrogels was determined according to the methodology described by $\mathrm{Pal}$ and $\mathrm{Pal},{ }^{18}$ using the equation: Swelling degree $=\left[\left(M_{\mathrm{t}}-M_{0}\right) / M_{0}\right] \times 100$, where $M_{t}$ is the mass of swelled sample at time $t$, and $M_{0}$ is the initial mass of dry sample. All hydrogel samples were put in contact with $50 \mathrm{~mL}$ ultrapure water during $1,2,3,6,12,24$, and $48 \mathrm{~h}$. All swelling assays were carried out in triplicate.

\section{Moisture Content Analyses via Infrared Heating}

For determination of the moisture content, a moisture analyzer producing heating via an halogen lamp was used (Shimadzu; model MOC63U), equipped with a programmable timer, self-calibration, and temperature adjusting between $50^{\circ} \mathrm{C}$ and $200^{\circ} \mathrm{C}$. The parameters utilized were temperature of $60^{\circ} \mathrm{C}$ in "slow" mode.

\section{Silver Content Determination by X-ray Fluorescence Analyses}

The silver content of the hydrogels impregnated with silver sulfadiazine was determined using a EDXRF spectrophotometer from Shimadzu (model EDX-700), which comprised an Rh tube and a $\mathrm{Si}(\mathrm{Li})$ semiconductor detector. Voltage applied to the X-ray tube was of $50 \mathrm{kV}$, with $25 \%$ lag time in the detector. The spectra were gathered sequentially, with a resolution of $0.02 \mathrm{keV}$, from 0 to $40 \mathrm{keV}$. The irradiation time was set at $120 \mathrm{~s}$.

\section{Tomographic Analyses via X-ray Transmission}

The tomographic images were gathered using a third generation X-ray transmission tomograph. ${ }^{19}$ The hydrogel samples 
were placed inside the tomograph chamber, and image slices were gathered using the following configurations of the tomographic system: operating voltage set at $29 \mathrm{kV}$ and electric current with $415 \mu \mathrm{A}$. The technique employed for obtaining the tomographic image involved acquisition of a large number of radiographs of the object (image slices), obtained by measuring the intensity of X-rays transmitted through the hydrogel sample, at different angular positions. The hydrogel samples were rotated $180^{\circ}$, with angular increments of $1^{\circ}$, producing 180 radiographs (projections) per image, each containing $652 \times 652$ pixels with a spatial resolution of $15.85 \mu \mathrm{m}$. At the outlet of the $\mathrm{X}$-ray source, one utilized an Al filter with $0.25 \mathrm{~mm}$ thickness. Appropriate mathematical algorithms were then used to reconstruct the 3D tomographic images of the hydrogel samples, through the appropriate composition of bi-dimensional (2D) images. The $3 \mathrm{D}$ images possessed $652 \times 652 \times 652$ pixels and the same spatial resolution of the $2 \mathrm{D}$ images, and thus the volume of data generated for each hydrogel sample is isotropic with relation to the spatial resolution. Having all the projections (radiographs gathered at each angular position), one utilized the software NRecon ${ }^{\mathrm{TM}}$ from Bruker microCT (version 1.6.9.4), which uses the algorithm of Feldkamp et al. ${ }^{20}$ in the process of reconstructing the tomographic images.

\section{Evaluation of Antimicrobial Activity of the Hydrogels via the Agar Diffusion Method}

The antimicrobial efficacy of the hydrogels integrating silver sulfadiazine was determined by applying the agar diffusion technique for susceptibility to antimicrobials described by Bauer et al. ${ }^{21}$ and Jorgensen and Ferraro, ${ }^{22}$ as well as according to the standards of the Clinical and Laboratory Standards Institute. ${ }^{23}$ The assays were carried out in duplicate using a strain of Staphylococcus aureus (CCCD S007 - Cefar Diagnóstico Ltda, São Paulo, Brazil). The Staphylococcus aureus strain was, initially, inoculated in BHI nutritive broth (Brain Heart Infusion) and maintained at $37 \pm 0.5^{\circ} \mathrm{C}$ during $24 \mathrm{~h}$, to allow growth of the bacteria. Following this time period, the bacterial culture was inoculated by spreading on two Petri dishes containing mannitol salt agar as culture medium, and the samples to be tested were then applied on the inoculated medium, in duplicate, with the aid of sterile tweezers near a Bunsen burner flame: hydrogel discs with silver sulfadiazine ( $\sim 7.0 \mathrm{~mm}$ in diameter and average weight of $8.00 \pm 0.01 \mathrm{mg})$, the negative standard of inhibition (hydrogel disc without silver sulfadiazine, with $\sim 7.0 \mathrm{~mm}$ in diameter), and the positive standard of inhibition [10 $\mathrm{mg}$ of reference ointment of silver sulfadiazine at $1 \%(\mathrm{w} / \mathrm{w})$ (with tradename Dermazine $\left.\left.{ }^{\circledR}\right)\right]$. The Petri dishes were then inverted and incubated under aerobic conditions at $37 \pm 0.5^{\circ} \mathrm{C}$ during $24 \mathrm{~h}$. After this time period, the plates were visually inspected for observation (or not) of any growth inhibition halos.

\section{Study of the Kinetics of Drug Release (Bioavailability) from the Hydrogel}

The drug release experiments were carried out by measuring the potential difference in an aqueous medium where a portion of hydrogel-containing silver sulfadiazine was immersed, using a combined silver electrode (model 9157; ThermoScientific Orion, San José, California) connected to a millivoltmeter (model pH300; pH analyzer from Analyser Instrumentação Analítica, São Paulo, Brazil). Initially, one tested magnetic stirring of the aqueous medium with the immersed hydrogel sample, as measures involving specific electrodes require homogenization of the reaction medium. However, the magnetic stirring promoted a complete (and accelerated) dissolution of the hydrogel. Hence, one opted to integrate a peristaltic pump, for continuous recirculation of the medium without promoting turbulent flow conditions. The peristaltic pump used (model MP13GJ-4; Ismatec, Glattbrugg, Switzerland) was connected to Tygon tubing (2.54 mm internal diameter). A weight sample of hydrogel of $0.2030 \mathrm{~g}$ [containing $\sim 1 \%$ (w/w) silver sulfadiazine] was immersed in $20 \mathrm{~mL}$ ultrapure water at room temperature $\left(25 \pm 0.1^{\circ} \mathrm{C}\right)$. Potential difference of the medium where the hydrogel was immersed was gathered at predetermined intervals of time, using the aforementioned combined silver electrode connected to a millivoltmeter. All measurements were gathered in $\mathrm{mV}$ by time of immersion of the hydrogel. The experiments were terminated when a silver sulfadiazine concentration plateau was reached.

\section{RESULTS AND DISCUSSION}

In the hydrogel formulations proposed in the research effort described herein, combinations of PVA with several degrees of hydrolysis, dextran, and silver sulfadiazine at $1 \%(\mathrm{w} / \mathrm{w})$ were evaluated. The concentration of $1 \%(\mathrm{w} / \mathrm{w})$ of silver sulfadiazine was chosen because of both its consensus in the treatment of burns and to the fact that commercial pharmaceutical formulations present such concentration. PVA and dextran were the polymers chosen to be utilized in the preparation of suitable hydrogels, as they present good characteristics for producing films. ${ }^{24}$ In a review paper by Baker et al., ${ }^{25}$ these researchers claim that it is possible to produce soft hydrogels with approximately $10 \%(\mathrm{w} / \mathrm{w})$ PVA and rigid hydrogels with approximately $50 \%-60 \%(\mathrm{w} / \mathrm{w})$ PVA. The major purpose of the research effort described herein was to produce a hydrogel with malleability, although with a certain resistance so that it could be utilized for topical applications. With this in mind, one tested formulations encompassing up to $24.5 \%$ (w/w) of polymers. PVA solubility increases with decreasing degree of hydrolysis. Thus, by mixing PVA polymers with different degrees of hydrolysis, it is possible to modulate the properties of both solubility and swelling of PVA and, consequently, release of the drug. ${ }^{26}$ The cross-linking process via cryogellification produced nonuniform hydrogels with uneven distribution of silver sulfadiazine, and thus one opted by using the chemical cross-linking process with glutaraldehyde. The hydrogels were analyzed with respect to homogeneity, resistance to manipulation, and color homogeneity. Formulations I, II, III, V, VI, VII, and VIII (see Table 1), chemically cross-linked with glutaraldehyde, were selected for the swelling and mechanical resistance tests.

\section{Swelling Degree}

Formulations VII and VIII were totally dissolved before completing $1 \mathrm{~h}$ of testing, and so they were discarded from further testing, by proving to be nonappropriate for using as wound bandages. From all the formulations tested (see Table 1), hydrogel formulations II, III, and V produced the best results (see Table 2). From inspection of Table 2, it can be observed that a maximum swelling occurred during the first hours of contact with ultrapure water (up to $6 \mathrm{~h}$ ). After this time period, the hydrogels began to lose mass, probably because of dissolution 
Table 2. Average Swelling Degrees and Associated Standard Deviations $(n=3)$ of Selected Hydrogel Formulations Following Immersion in Water for $1,3,6,12,24$, and $48 \mathrm{~h}$

\begin{tabular}{lccc}
$\begin{array}{l}\text { Immersion } \\
\text { Time }(\mathrm{h})\end{array}$ & $\begin{array}{c}\text { Formulation II } \\
(\overline{\%} \pm \sigma)\end{array}$ & $\begin{array}{c}\text { Formulation III } \\
(\overline{\%} \pm \sigma)\end{array}$ & $\begin{array}{c}\text { Formulation V } \\
(\overline{\%} \pm \sigma)\end{array}$ \\
\hline 1 & $200.40 \pm 3.88$ & $190.00 \pm 1.56$ & $202.50 \pm 5.09$ \\
3 & $210.56 \pm 2.42$ & $220.00 \pm 0.87$ & $234.20 \pm 2.39$ \\
6 & $232.61 \pm 0.73$ & $266.00 \pm 0.81$ & $225.99 \pm 1.85$ \\
12 & $179.42 \pm 0.53$ & $197.15 \pm 2.20$ & $186.54 \pm 2.56$ \\
24 & $128.71 \pm 1.29$ & $194.32 \pm 3.16$ & $155.52 \pm 5.00$ \\
48 & $104.76 \pm 2.34$ & $172.03 \pm 3.01$ & $128.85 \pm 1.28$ \\
\hline
\end{tabular}

of the films. Such loss of mass was smaller for the hydrogel produced according to formulation III, and thus this formulation was selected for further work.

\section{Mechanical Resistance}

To determine the mechanical properties of the selected hydrogels, these were evaluated with respect to perforation, relaxation, and resilience. The mechanical properties of the hydrogel films are mainly related with the polymer's ability to form bonds in polymer chains, making their separation difficult when subject to mechanical forces. ${ }^{27}$ The plasticizer used also has an influence on these properties. ${ }^{28}$ The analysis of the results considered the average thickness $(0.50 \pm 0.03 \mathrm{~mm})$ and the average weight ( $344.18 \pm 2.65 \mathrm{mg})$ of the hydrogels with an area of 25 $\times 30 \mathrm{~mm}^{2}$. The results produced during evaluation of the mechanical resistance of selected hydrogel formulations with and without silver sulfadiazine, with respect to perforation, relaxation, and resilience are displayed in Table 3.

Hydrogel formulations II and V, containing in their composition a higher amount of PVA99 $(15 \%$, w/w, of the formulation), exhibited a higher resistance than the hydrogel produced according to formulation III, which contained only $10 \%(\mathrm{w} / \mathrm{w})$ PVA99 in its composition. The hydrogel produced according to formulation II, incorporating silver sulfadiazine, exhibited a lower resistance to perforation than its counterpart without silver sulfadiazine; however, it presented higher values relative to resilience and relaxation. Hence, it may be concluded that the presence of silver sulfadiazine improved its viscoelastic characteristics of deformation and molecular relaxation. $\mathrm{Hy}$ drogels produced according to formulations III and V presented a slight decrease in the mechanical resistance for the parameters analyzed, except for the hydrogel produced according to formulation $\mathrm{V}$ with silver sulfadiazine, which presented a better mechanical resistance in relation to resilience than its counter- part without silver sulfadiazine. The major goal of the research work undertaken was to develop a hydrogel suitable for application in skin wounds. In our perspective, a hydrogel bandage applied directly to a skin wound that produces exudate should not strongly adhere to the wound, because its exchange would become unnecessary painful to the patient and would not allow re-epithelialization of the wound. Thus, perforation, relaxation, and resilience were the mechanical resistance parameters evaluated, whereas adhesiveness or bioadhesiveness were not at all considered important in the research work developed. The optimized hydrogel is intended to be applied to a skin wound and maintained in place by a bandage, and not to stick or adhere to the wounded skin. Our perspective is supported by literature references. ${ }^{29,30}$ Hydrogels act as moist wound dressing materials, and are able to both absorb and retain the wound exudates as well as any contaminating bodies such as bacteria, within their network structure. Additionally, hydrogels have been found to both promote fibroblast proliferation by reducing fluid loss from the wound surface and protect the wound from external noxae, sine qua non conditions necessary for a rapid wound healing. ${ }^{30}$ Hydrogels also help in maintaining a suitable microclimate on the wound surface, of utmost importance for the biosynthetic reactions necessary for cellular activities. Proliferation of fibroblasts is necessary for a complete re-epithelialization of the wound, which starts from its edge. As hydrogels help to maintain the wound moist, keratinocytes can thus migrate on its surface. ${ }^{30}$ Hence, hydrogel-based dressings are of undeniable value in a wide spectrum of wounds, and have the potential to be utilized on necrotic, sloughy, granulating, and epithelializing wounds, and can also be applied in infected wounds, provided that the patient is being treated with systemic antibiotics and the dressing is changed on a daily basis. In their work, Rippon et al. ${ }^{29}$ also describe several wound dressing definitions, to include (1) adherent dressings (dressings that are likely to adhere to any type of drying wound, viz., cotton gauze and/or simple dressing pads), (2) low-adherent dressings (dressings with a wound contact surface that is designed to reduce adherence, viz., absorbent wound dressings), (3) nonadherent dressings (dressings that maintain a moist gel layer over the wound that is not expected to adhere, provided that it is not allowed to dry out, viz., alginates/hydrocolloids and/or hydrogels/hydrofibres), and (4) atraumatic dressings (dressings that do not cause trauma either to the wound or the peri-wound skin upon removal, viz., soft silicone dressings). Clearly, the hydrogels developed, optimized, and fully characterized in the research word described herein, fall in category (3) of the aforementioned descriptions.

Table 3. Results from the Mechanical Resistance Tests $(\mathrm{kg} \pm \sigma)$ Performed to Selected Hydrogels, Relative to Perforation, Relaxation, and Resilience ( $\mathrm{s} \pm \sigma)$, as a Function of Applied Force (kg) (AUC)

\begin{tabular}{|c|c|c|c|c|c|c|}
\hline \multirow[b]{2}{*}{ Hydrogel Formulation } & \multicolumn{2}{|c|}{ Perforation } & \multicolumn{2}{|c|}{ Relaxation } & \multicolumn{2}{|c|}{ Resilience } \\
\hline & Force (kg) & $\operatorname{AUC}(\mathrm{kg} / \mathrm{s})$ & Force $(\mathrm{kg})$ & $\mathrm{AUC}(\mathrm{kg} / \mathrm{s})$ & Force (kg) & $\mathrm{AUC}(\mathrm{kg} / \mathrm{s})$ \\
\hline II & $2.571 \pm 0.320$ & $7.445 \pm 1.433$ & $0.439 \pm 0.072$ & $0.630 \pm 0.095$ & $0.352 \pm 0.082$ & $0.498 \pm 0.135$ \\
\hline II w/SDZ & $0.781 \pm 0.165$ & $3.351 \pm 0.225$ & $0.690 \pm 0.032$ & $2.974 \pm 0.790$ & $0.136 \pm 0.037$ & $1.880 \pm 0.020$ \\
\hline III & $0.791 \pm 0.162$ & $0.944 \pm 0.269$ & $0.258 \pm 0.027$ & $0.355 \pm 0.039$ & $0.335 \pm 0.020$ & $0.439 \pm 0.335$ \\
\hline III w/SDZ & $0.765 \pm 0.234$ & $0.915 \pm 0.279$ & $0.215 \pm 0.043$ & $0.303 \pm 0.104$ & $0.193 \pm 0.005$ & $0.514 \pm 0.032$ \\
\hline $\mathrm{V}$ & $1.373 \pm 0.063$ & $2.224 \pm 0.190$ & $0.224 \pm 0.027$ & $0.298 \pm 0.056$ & $0.252 \pm 0.017$ & $0.535 \pm 0.042$ \\
\hline $\mathrm{V}$ w/SDZ & $0.862 \pm 0.181$ & $3.134 \pm 0.077$ & $0.456 \pm 0.054$ & $0.580 \pm 0.032$ & $0.688 \pm 0.076$ & $1.926 \pm 0.040$ \\
\hline
\end{tabular}

$n=3$.

AUC, area under the curve; SDZ, silver sulfadiazine. 
Wound dressings containing silver may be utilized in acute wounds, such as those produced by burns or surgery and in chronic wounds with infections. The major functions of antimicrobial dressings, such as those containing silver, involve reducing the microbial load in acute or chronic wounds, or act as an antimicrobial barrier for wounds with high risk of infection or reinfection. ${ }^{31}$ Additionally, dressings allow the creation of a moist and warm environment surrounding the wound, thus bringing benefits to the healing process. ${ }^{32}$ The developed product presents low adhesion to the injured tissue, which is a desirable feature, as adherent dressings may cause pain and even more tissue damage during removal. ${ }^{32}$ However, their fixing requires the use of natural or synthetic bandages (cotton wool, lint, and gauzes). Because of the product characteristics, this will virtually not harm the performance of the hydrogel. Hence, hydrogel adhesion to bandage materials will not be a problem. The developed hydrogels possess PVC in their composition, which is a hydrophobic polymer with a low-surface free energy $^{33}$ that do not favor attraction of the water molecules present in the dispersion containing the polymers. As a consequence, this promotes the nonadherence of the hydrogel to the bandages and healings that may be utilized.

\section{Structural Microanalysis of Selected Hydrogels Incorporating Silver Sulfadiazine via SEM}

From the results gathered in the SEM analyses performed to the selected hydrogel formulation, namely, formulation III, a uniform morphology could be observed, with points of deposition of silver sulfadiazine clearly seen in the micrographs (see Fig. 1) at several magnifications. The polymeric structures displayed by the hydrogel formulation facilitate solvation and dissolution upon contact with the solvent, skin moisture, and with the skin burn, thus being able to slowly release its silver sulfadiazine content. The hydrogel micrographs in Figure 1 were obtained via the EBSD (electron backscatter diffraction) technique, which allows the crystallographic analysis of the material, and it can be observed that the hydrogel topography is highly uniform.

\section{FTIR Analyses}

Fourier transform infrared spectrophotometric analyses allow identification of functional groups. Each functional group in particular absorbs radiation in a characteristic frequency of the infrared spectrum. Thus, a plot of intensity versus radiation frequency, known as infrared spectrum, allows characterization of the functional groups in a given material, ${ }^{35}$ which makes FTIR spectrophotometry quite used in the analysis of the structure of polymeric systems. This analytical technique may allow clarification of possible interactions between the incorporated drug and the polymeric matrix of the hydrogel, through the analysis of the functional groups of the agents involved in the process. The infrared spectrum of pure silver sulfadiazine (1) and the infrared spectra of the hydrogels produced according to formulation III, without (2) and with (3) silver sulfadiazine, as well as, are displayed in Figure 2. Comparing the spectrum of silver sulfadiazine (see Fig. 2a) with the spectrum of the hydrogel produced according to formulation III with incorporated silver sulfadiazine (see Fig. 2c), it can be observed that the characteristic peaks of silver sulfadiazine in the region from 1300 to $1100 \mathrm{~cm}^{-1}$ are present in the hydrogel containing silver sulfadiazine (see Fig. 2c) but with reduction in peak intensity. This
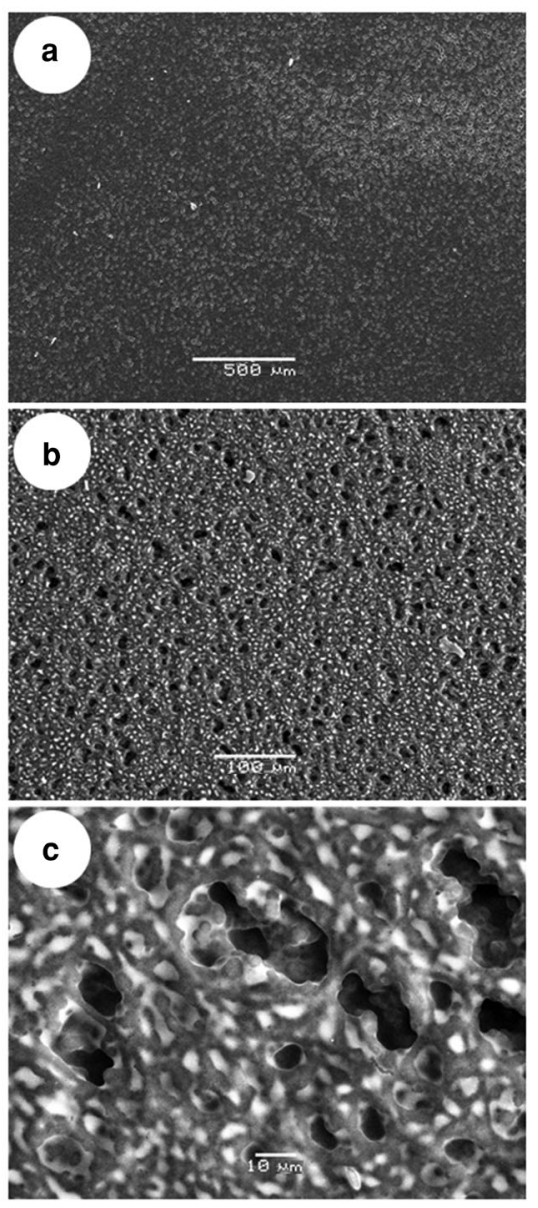

Figure 1. Scanning electron micrograph of a hydrogel of PVA with incorporated silver sulfadiazine (formulation III), gathered using the electron back-scattered diffraction technique, at different magnifications: (a) $50 \times$; (b) $200 \times$; (c) $1000 \times$.

clearly suggests that the chemical aspect of the drug was preserved during production of the hydrogel, allowing to conclude that silver sulfadiazine did not engage in any chemical interaction with the hydrogel components, only being carried by the hydrogel, which otherwise could have reduced its antimicrobial activity.

\section{XRD Analyses}

According to Costa Jr. and Mansur, ${ }^{34}$ the XRD analysis of a blend of PVA/chitosan revealed that PVA presents an amorphous form. The results obtained from the XRD analyses performed to selected hydrogels are displayed in Figure 3 in the form of normalized diffractograms, for the selected hydrogel prepared according to formulation III with (see Fig. 3b) and without (see Fig. 3c) silver sulfadiazine, and allows to observe an amorphous behavior without peaks of crystallinity. The small peaks at $2 \theta 41^{\circ}$ are related with the presence of residual water in the samples. These results corroborate the results obtained via SEM, which indicated the formation of an amorphous film. The decisive advantage of the methods of analysis by XRD over other analytical techniques is based on the uniqueness of the diffraction patterns of crystalline substances, on the ability to distinguish between elements and their oxides, and on 

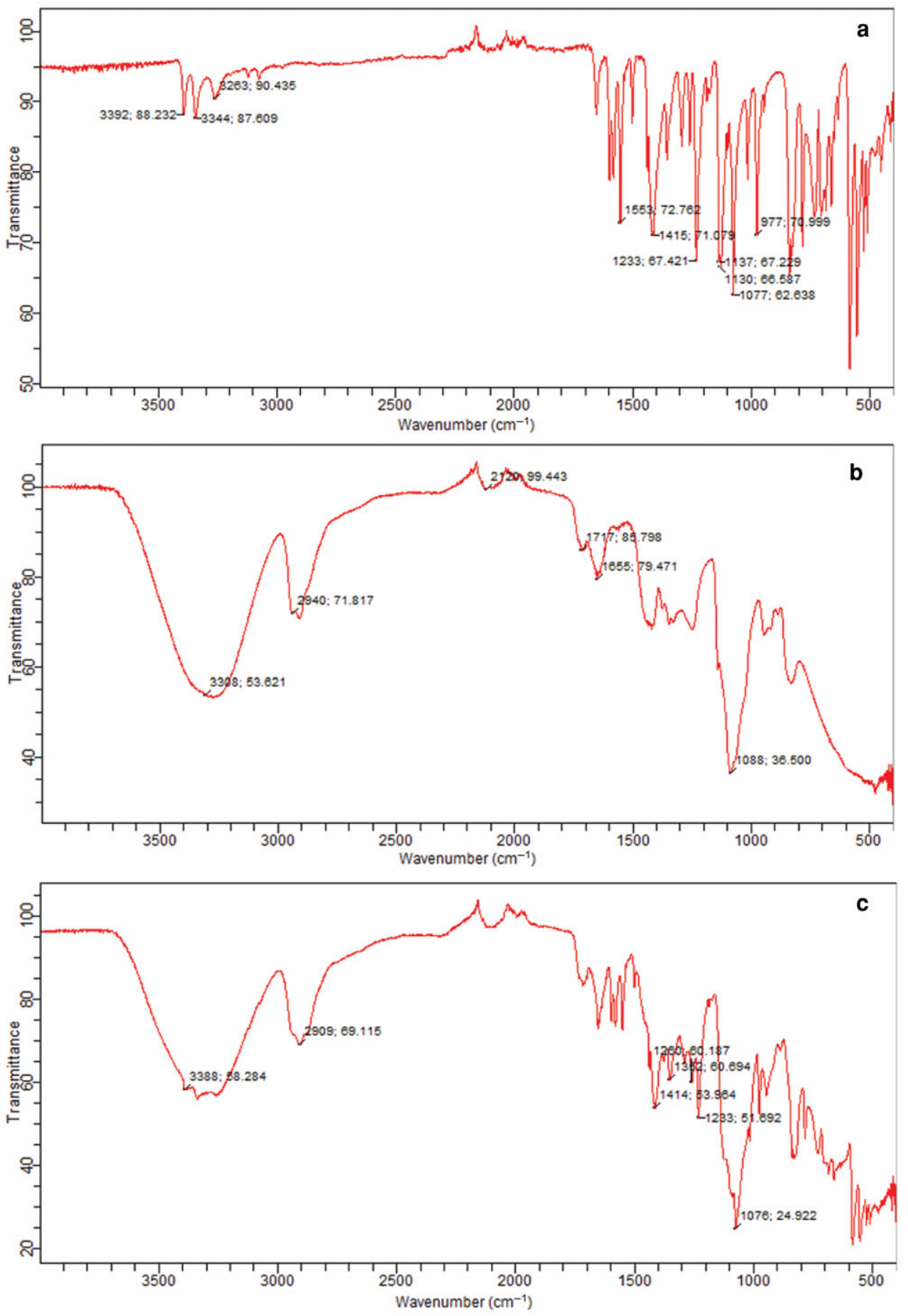

Figure 2. Fourier transform infrared spectra of silver sulfadiazine (a), hydrogel produced according to formulation III without silver sulfadiazine (b), and hydrogel produced according to formulation III with silver sulfadiazine (c). The FTIR spectra were gathered in the region of wavelengths from 4000 to $400 \mathrm{~cm}^{-1}$ with resolution of $2 \mathrm{~cm}^{-1}$, in an FTIR spectrophotometer from Agilent (model Cary 630).

the possibility of identifying chemical compounds, polymorphic forms, and mixed crystals, via nondestructive analyses.

Peaks having high intensity and narrow base width are related to crystalline materials, whereas wide base peaks are related to amorphous materials. As the intensity of peaks in the XRD patterns also depends on the concentration of the active drug, an increase in both the intensity of peaks and diffraction pattern was expected for the hydrogel impregnated with sil- ver sulfadiazine (see Fig. 3b) relative to its plain counterpart (without silver sulfadiazine, see Fig. 3c). Both diffractograms present the same intensity pattern, but with different amplitudes in several peaks. Normalization of intensity in all diffractograms was performed by dividing the intensity values by the maximum intensity value in each diffractogram, thus allowing a better comparison between the X-ray diffractograms of the two hydrogels. 


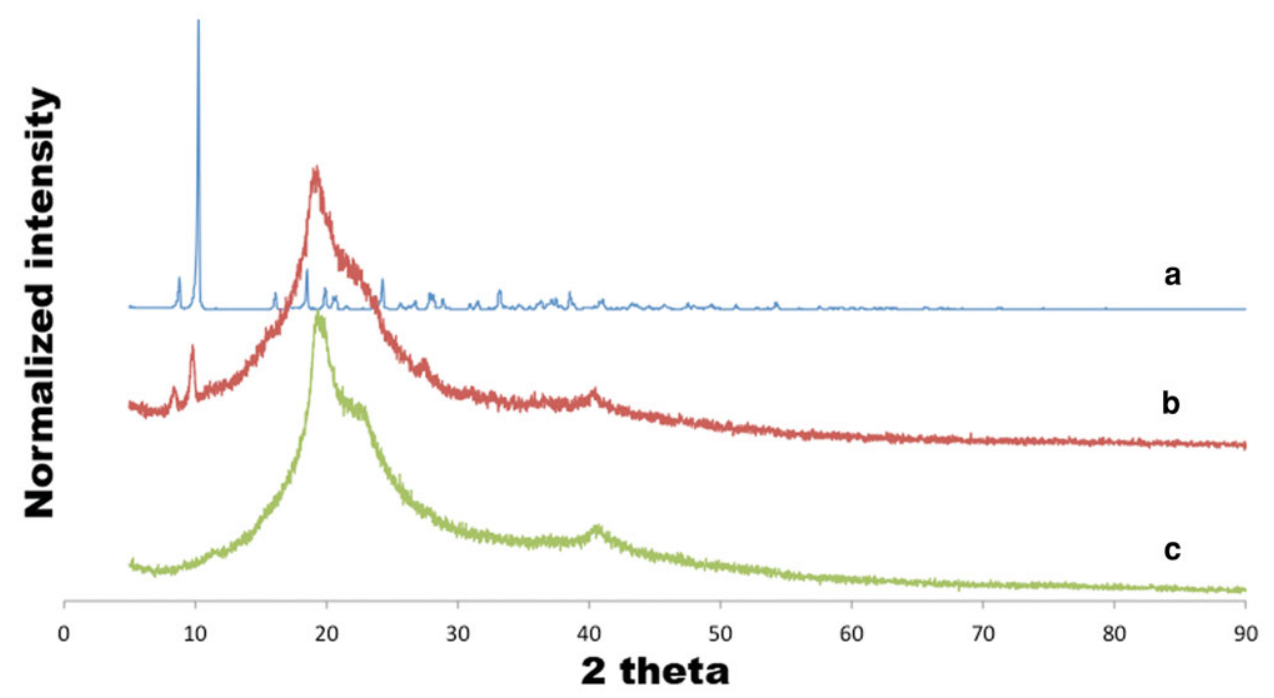

Figure 3. X-ray diffractograms of samples of silver sulfadiazine (a) and of samples of hydrogel prepared according to formulation III with silver sulfadiazine (b), and of hydrogel prepared according to formulation III without silver sulfadiazine (c). The X-ray diffractograms were gathered using $\mathrm{Cu}$ target-filtered X-rays. The analyses were carried out at diffraction angles of $2-\theta$ (from $5^{\circ}$ to $90^{\circ}$, with increments of $0.02^{\circ}$ and rate of $2 \% \mathrm{~min}^{-1}$ ) with voltage of $40 \mathrm{kV}$, current intensity of $30 \mathrm{~mA}$, and X-ray power of $3 \mathrm{~kW}$, in an X-ray diffractometer from Shimadzu (model XRD7000).

\section{X-ray Transmission Tomography}

From the tomographic analyses via X-ray transmission performed to the hydrogel prepared according to formulation III with impregnated silver sulfadiazine (see Fig. 4), it can be observed that silver sulfadiazine migrate to the surface of the hydrogel (purple-bluish layer; see Figs. 4a and 4b) during the polymerization process. Because of absorbing more radiation and of its higher atomic density, silver is thus in greater evidence as highlighted in the tomographic images displayed as Figure 4 . These results are in close agreement with those obtained by infrared spectrophotometry that indicated that silver sulfadiazine probably did not engage in any chemical bonding with the polymeric matrix. This is clearly an important and positive data, as by not engaging in any chemical bonding with the polymeric surface, silver sulfadiazine becomes readily available and maintains its antimicrobial activity.

Additionally, as can be seen from inspection of the data in Table 4, the layer of silver sulfadiazine (in purple-bluish in Fig. 4) is significantly less porous than the polymeric matrix itself (in green in Fig. 4), and presented a volume 1.5 times smaller than that of the polymeric film. Anisotropy is the property of being directionally dependent, as opposed to isotropy, which implies identical properties in all directions. Anisotropy can thus be defined as a difference, when measured along different axes, in a material's physical or mechanical properties (e.g., absorbance). The degree of anisotropy, calculated as Degree of anisotropy $=\left[1-\left(\frac{\text { min Eigenvalue }}{\text { max Eigenvalue }}\right)\right]$, is 0 for total isotropy and 1 for total anisotropy. Hence, as can be seen from inspection of the data in Table 4, the degree of anisotropy is 0.562 and 0.623 for the layer of silver sulfadiazine (in purplebluish in Fig. 4) and the polymeric matrix (in green in Fig. 4), respectively, values that are half-way in the aforementioned scale. When analyzing the structure of these two interconnecting regions, one finds that the layer of silver sulfadiazine has a lower number of closed pores (viz., 215) than that of the polymeric matrix (viz., 356), corresponding to an open porosity of $18.54 \%$

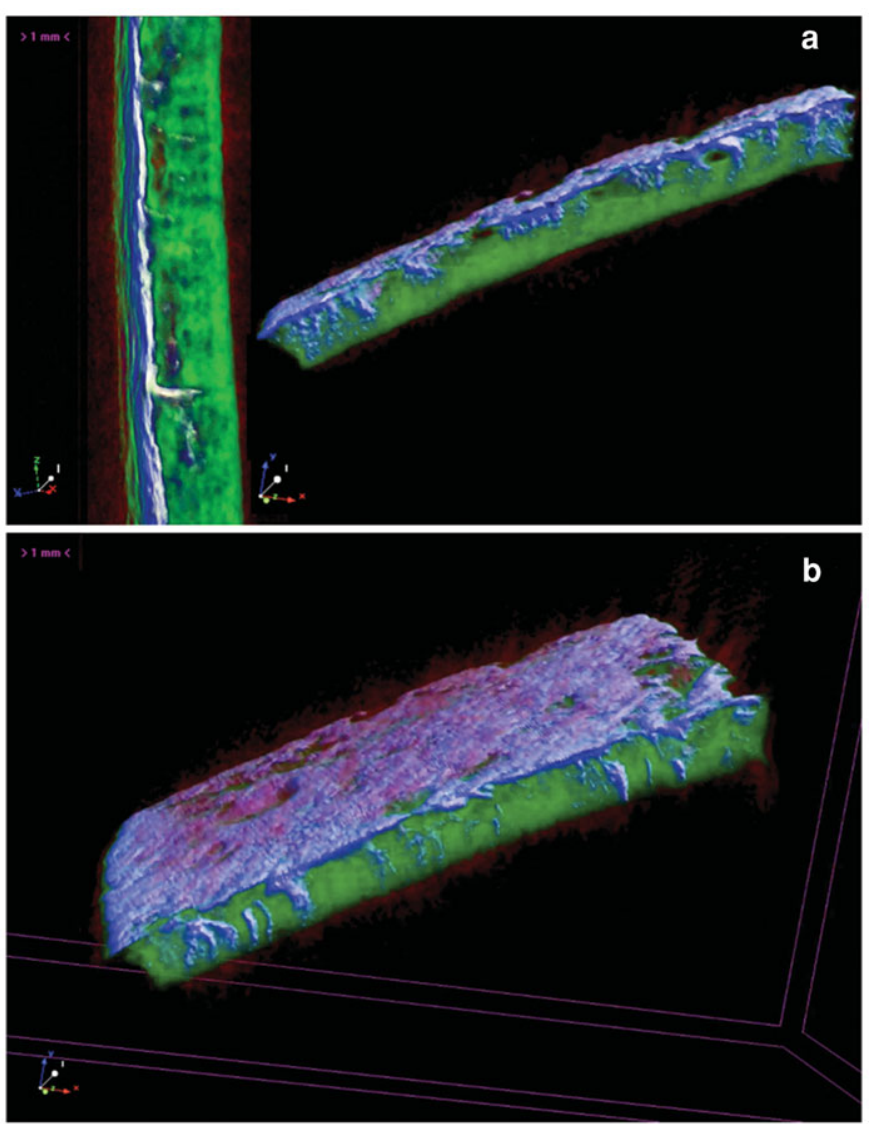

Figure 4. Images obtained by tomographic analyses via X-ray transmission of the hydrogel prepared according to formulation III, with impregnated silver sulfadiazine, being (a) a cross-cut profile image of the hydrogel with silver sulfadiazine, and (b) slant profile image of the hydrogel with impregnated silver sulfadiazine. Three-dimensional image slices were gathered using an operating voltage set at $29 \mathrm{kV}$ and electric current with $415 \mu \mathrm{A}$ 
Table 4. Results Obtained from the Tomographic Analyses via X-ray Transmission Performed to the Hydrogel Prepared According to Formulation III with Impregnated Silver Sulfadiazine

\begin{tabular}{|c|c|c|}
\hline \multirow[b]{2}{*}{ Parameter } & \multicolumn{2}{|c|}{ Porosity } \\
\hline & $\begin{array}{c}\text { Silver Sulfadiazine Layer } \\
\text { (Purple-Bluish Layer in Fig. 4) }\end{array}$ & $\begin{array}{l}\text { Polymeric Matrix Without } \\
\text { Silver Sulfadiazine (Green Part in Fig. 4) }\end{array}$ \\
\hline Number of layers & 79 & 79 \\
\hline Pixel size & $15.85012 \mu \mathrm{m}$ & $15.85012 \mu \mathrm{m}$ \\
\hline Total VOI (volume of interest), TV & $0.59154 \mathrm{~mm}^{3}$ & $0.87446 \mathrm{~mm}^{3}$ \\
\hline Object volume, Obj.V & $0.48085 \mathrm{~mm}^{3}$ & $0.58278 \mathrm{~mm}^{3}$ \\
\hline Percent object volume, Obj.V/TV & $81.28675 \%$ & $66.64513 \%$ \\
\hline Total VOI surface, TS & $15.15924 \mathrm{~mm}^{2}$ & $22.44291 \mathrm{~mm}^{2}$ \\
\hline Object surface, Obj.S & $24.91102 \mathrm{~mm}^{2}$ & $52.14039 \mathrm{~mm}^{2}$ \\
\hline Intersection surface, i.S & $8.23326 \mathrm{~mm}^{2}$ & $9.31923 \mathrm{~mm}^{2}$ \\
\hline Object surface-volume ratio, Obj.S-Obj.V & $51.80672 \mathrm{~mm}^{-1}$ & $89.46802 \mathrm{~mm}^{-1}$ \\
\hline Object surface density, Obj.S/TV & $42.11200 \mathrm{~mm}^{-1}$ & $59.62608 \mathrm{~mm}^{-1}$ \\
\hline Fragmentation index, Fr.I & $-22.89647 \mathrm{~mm}^{-1}$ & $-52.01265 \mathrm{~mm}^{-1}$ \\
\hline Degree of anisotropy, DA & $2.28535(0.56243)$ & $2.65099(0.62278)$ \\
\hline Eigenvalue 1 & 0.00787 & 0.02925 \\
\hline Eigenvalue 2 & 0.01093 & 0.06543 \\
\hline Eigenvalue 3 & 0.01800 & 0.07754 \\
\hline Number of closed pores, Po.N(cl) & 215 & 356 \\
\hline Volume of closed pores, Po.V(cl) & $0.00100 \mathrm{~mm}^{3}$ & $0.00176 \mathrm{~mm}^{3}$ \\
\hline Surface of closed pores, Po.S(cl) & $0.34265 \mathrm{~mm}^{2}$ & $0.60716 \mathrm{~mm}^{2}$ \\
\hline Closed porosity (percent), $\mathrm{Po}(\mathrm{cl})$ & $0.20785 \%$ & $0.30037 \%$ \\
\hline Volume of open pore space, Po.V(op) & $0.10970 \mathrm{~mm}^{3}$ & $0.28992 \mathrm{~mm}^{3}$ \\
\hline Open porosity (percent), $\mathrm{Po}(\mathrm{op})$ & $18.54394 \%$ & $33.15408 \%$ \\
\hline Total volume of pore space, Po.V(tot) & $0.11070 \mathrm{~mm}^{3}$ & $0.29167 \mathrm{~mm}^{3}$ \\
\hline Total porosity (percent), Po(tot) & $18.71325 \%$ & $33.35487 \%$ \\
\hline Euler number, Eu.N & -1284 & -5615 \\
\hline Connectivity, Conn & 1694 & 6435 \\
\hline Connectivity density, Conn.Dn & $2863.70196 \mathrm{~mm}^{-3}$ & $7358.85996 \mathrm{~mm}^{-3}$ \\
\hline
\end{tabular}

and a total porosity of $18.71 \%$ for the silver sulfadiazine layer and an open porosity of $33.15 \%$ and a total porosity of $33.35 \%$ for the polymeric matrix. Additionally, the fragmentation index (see Table 4) gives an index of connectivity and calculates an index of relative convexity or concavity of the total object surface, on the principle that concavity indicates connectivity (and the presence of "nodes"), and convexity indicates isolated disconnected structures (struts). Lower fragmentation indexes signify better connected lattices, whereas higher fragmentation indexes mean a more disconnected structure. The prevalence of enclosed cavities and concave surfaces can push the fragmentation index to negative values, which was in fact determined in the analyses performed (see Table 4). These results are quite important because of the fact that silver sulfadiazine migrates to one of the surfaces of the polymeric structure during the polymerization process; the hydrogel can be applied in two different ways, namely, with silver sulfadiazine layer toward the skin or with the silver sulfadiazine layer opposite from the skin. If the hydrogel is applied with the silver sulfadiazine toward the skin, a lower total porosity will make the active principle readily available to the skin lesion. On the contrary, if the hydrogel is applied with the silver sulfadiazine layer opposite from the skin, a higher porosity is important to allow swelling of the polymeric matrix and concomitant (slow) release of the active principle to the skin lesion.

\section{Moisture Content}

Selected hydrogels without inclusion of the active principle (silver sulfadiazine) presented a moisture content between $11.35 \%$
Table 5. Results from Moisture Content (\%, Average and Associated Standard Deviation) Determinations of Selected Hydrogel Samples ( $n$ =3) Using a Moisture Infrared Analyzer

\begin{tabular}{lc}
\hline Hydrogel Formulation & Moisture Content $(\overline{\%} \pm \sigma)$ \\
\hline II & $12.53 \pm 0.15$ \\
II w/SDZ & $10.74 \pm 0.08$ \\
III & $11.47 \pm 0.17$ \\
III w/SDZ & $10.59 \pm 0.06$ \\
V & $11.35 \pm 0.10$ \\
V w/SDZ & $10.93 \pm 0.06$ \\
\hline
\end{tabular}

(w/w) and $12.53 \%(\mathrm{w} / \mathrm{w})$, whereas the same hydrogels impregnated with silver sulfadiazine presented a moisture content between $10.74 \%(\mathrm{w} / \mathrm{w}$ ) and $10.93 \%$ (w/w) (see Table 5). These results may be an indication that the drying process utilized allowed elimination of almost all the free water except, probably, for that water bound to the polymeric matrix. Considering the low moisture contents determined, the hydrogel developed shows a promising characteristic for storage.

In a recent work by Vila et al., ${ }^{36}$ a hydrogel of PVA was developed, integrating nitrofurazone, with a moisture content of approximately $18 \%$ (w/w). In another (recent) work by Vila et al., ${ }^{37}$ a fast dissolution film for buccal applications, made of the polymer pullulan, presented a moisture content of approximately $14 \%(\mathrm{w} / \mathrm{w})$. In the present research work, the moisture contents of the hydrogels produced were lower (between $11.35 \%$, w/w, and $12.53 \%$, w/w), indicating that the drying 
Table 6. Average Values and Associated Standard Deviations $(n=3)$ of the Silver Contents (ppm) in Selected Hydrogels Obtained by Energy-Dispersion X-ray Fluorescence Analyses $(n=3)$

\begin{tabular}{lccc}
\hline $\begin{array}{l}\text { Hydrogel } \\
\text { Formulation }\end{array}$ & $\begin{array}{c}\text { Silver } \\
\text { Content }(\mathrm{ppm})\end{array}$ & $\begin{array}{c}\text { Silver } \\
\text { Content }(\%)\end{array}$ & $\begin{array}{c}\text { Silver Sulfadiazine } \\
\text { Content }(\%)\end{array}$ \\
\hline II & $301.00 \pm 10.00$ & 0.03010 & 1.030 \\
III & $325.50 \pm 15.00$ & 0.03255 & 1.085 \\
V & $384.50 \pm 94.00$ & 0.03845 & 1.280 \\
\hline
\end{tabular}

process utilized was efficient to remove the water, producing dried hydrogels that can be stored for long periods of time because of their relatively low-moisture content.

\section{Silver Content Determination by X-ray Fluorescence Analyses}

Table 6 displays the results (average and standard deviation) of silver content in selected hydrogels, obtained by EDXRF. All analyses were carried out in triplicate. The hydrogels were prepared so as to present $1 \%(\mathrm{w} / \mathrm{w})$ silver sulfadiazine. The molecular weight of silver sulfadiazine is $358.4 \mathrm{~g} / \mathrm{mol}$, whereas that of silver is $107.86 \mathrm{~g} / \mathrm{mol}$. Thus, in a molecule of silver sulfadiazine, $30 \%$ of the weight corresponds to silver. When preparing $100 \mathrm{~mL}$ of hydrogel formulation, one utilized $0.1 \mathrm{~g}$ of silver sulfadiazine $(1 \%$, w/w). Hence, in $0.1 \mathrm{~g}$ silver sulfadiazine, there is $0.030 \%$ silver. From the results obtained by EDXRF analyses, hydrogels prepared according to formulations II and III presented a silver sulfadiazine content within the expected value ( $1.030 \%$ and $1.085 \%$, respectively). For the hydrogel prepared according to formulation $\mathrm{V}$, the silver sulfadiazine content determined was slightly higher than expected $(1.280 \%, \mathrm{w} / \mathrm{w})$, probably because of an uneven distribution of silver sulfadiazine in the hydrogel.

\section{Antimicrobial Activity of the Hydrogel}

The hydrogel prepared according to formulation III, without the active principle (functioning as negative standard of inhibition), did not inhibit bacterial growth (no growth inhibition halo produced). The hydrogel film containing silver sulfadiazine ( $8 \mathrm{mg}$ of hydrogel film, containing $\sim 0.08 \mathrm{mg}$ silver sulfadiazine) promoted a microbial growth inhibition halo (diameter of $1.45 \mathrm{~cm})$ slightly higher $(\sim 1.54 \times)$ than that of the positive standard of inhibition (silver sulfadiazine ointment at $1 \%, \mathrm{w} / \mathrm{w}$, namely, $10 \mathrm{mg}$ Dermazine ${ }^{\circledR}$ containing $\sim 0.10 \mathrm{mg}$ silver sulfadiazine, halo diameter of $0.94 \mathrm{~cm}$ ), meaning that the hydrogel containing impregnated silver sulfadiazine allowed a continuous release of the antimicrobial principle, in concentrations able to inhibit bacterial growth.

\section{Thermal Analyses via TGA and DSC}

In general, thermal analyses are useful tools in developing formulations as they allow to evaluate the compatibility between components of a formulation and the stability and thermal decomposition of drugs. The data obtained by thermal analysis are directly related to the final quality of a pharmaceutical product, allowing to infer aspects of therapeutic efficacy of the product or the stability of the product throughout the shelf life (or validity) period. TGA accompany the variation in sample mass as a function of a linear increase in temperature during a predetermined time interval, allowing to determine the thermal profile of the hydrogels prepared. The TGA curves and the first derivatives of the mass loss curves for the hydrogels prepared according to formulation III are displayed in Figure 5, respectively, for the hydrogel without impregnated silver sulfadiazine (orange curves) and for the hydrogel with impregnated silver sulfadiazine (blue curves). As can be observed from inspection of the curves in Figure 5, the thermal behavior is quite similar in both hydrogels, with a small mass loss around approximately $100^{\circ} \mathrm{C}$ indicating loss of water. Around $250^{\circ} \mathrm{C}$, one can notice a significant loss of mass, indicating degradation of the components in the hydrogels. The first derivative of the weight loss curve (i.e., the rate of mass change; see Fig. 5) can be used to tell the points at which weight loss is most apparent (inflection points), namely, $60^{\circ} \mathrm{C}$ and $310^{\circ} \mathrm{C}$ for the hydrogel without silver sulfadiazine, and $65^{\circ} \mathrm{C}$ and $290^{\circ} \mathrm{C}$ for the hydrogel impregnated with silver sulfadiazine.

Thermal analysis by DSC is an analysis technique in which the temperature of a sample, compared with the temperature of a thermally inert material, is recorded as the sample is heated or cooled at a constant rate. ${ }^{35}$ The DSC technique of analysis thus measures the enthalpy of the samples, and may indicate the glass transition temperature and endothermic and/or exothermic events in the samples under scrutiny. The results of thermal analysis by DSC can be found in Figure 6. The hydrogel produced according to formulation III possesses in its composition $10 \%$ (w/w) PVA with a degree of hydrolysis of $89 \%$ and $10 \%$ (w/w) PVA with a degree of hydrolysis of $99 \%$. The thermogram obtained (see Fig. 6) presented two endothermic events, one at $128.13^{\circ} \mathrm{C}$ (hydrogel without silver sulfadiazine) and one at $128.66^{\circ} \mathrm{C}$ (hydrogel with silver sulfadiazine). The other endothermic events occurred at $218.13^{\circ} \mathrm{C}$ (hydrogel without silver sulfadiazine) and $215.67^{\circ} \mathrm{C}$ (hydrogel with silver sulfadiazine). The data obtained indicated that the components utilized, in general, only slightly influenced the thermal behavior of the hydrogels, and also that incorporation of silver sulfadiazine did not compromise the stability of the hydrogel for the intended use. The presence of silver sulfadiazine promoted a slight shift of the endothermic event close at $200^{\circ} \mathrm{C}$, from $218.13^{\circ} \mathrm{C}$ to $215.67^{\circ} \mathrm{C}$, leading to a better thermal stability of the hydrogel produced according to formulation III. Differential scanning calorimetric analyses of hydrogel formulations provided an insight into the state and degree of crystallinity, and the melting and crystallization behavior of crystalline materials. The addition (impregnation) of silver sulfadiazine promoted a slight increase in the melting temperature peak [absorption peak at $128.13^{\circ} \mathrm{C}$ and heat absorbed (melting enthalpy) of $340.6 \mathrm{~J} / \mathrm{g}$ for the control hydrogel (devoid of any active drug) formulation, to absorption peak at $\sim 128.66^{\circ} \mathrm{C}$ and heat absorbed (melting enthalpy) of $478.2 \mathrm{~J} / \mathrm{g}$ for the optimized hydrogel with impregnated silver sulfadiazine (see Fig. 6)], and slight decrease in the decomposition temperature peak [absorption peak at $218.13^{\circ} \mathrm{C}$ and heat absorbed (melting enthalpy) of $10.89 \mathrm{~J} / \mathrm{g}$ for the control hydrogel (devoid of any active drug) formulation, to absorption peak at $\sim 215.67^{\circ} \mathrm{C}$ and heat absorbed (melting enthalpy) of $5.018 \mathrm{~J} / \mathrm{g}$ for the optimized hydrogel with impregnated silver sulfadiazine (see Fig. 6)], denoting a narrowing of the melting temperature range in the case of the optimized hydrogel formulation.

Inclusion of silver sulfadiazine in the hydrogel led to a slight decrease in the peak of heat absorption $\left(215.67^{\circ} \mathrm{C}\right)$, when compared with its blank counterpart $\left(218.13^{\circ} \mathrm{C}\right)$, because of amorphization of the system. The results obtained from the DSC thermal analyses carried out with both silver sulfadiazineloaded and sulfadiazine-unloaded hydrogel (see Fig. 6) are in 

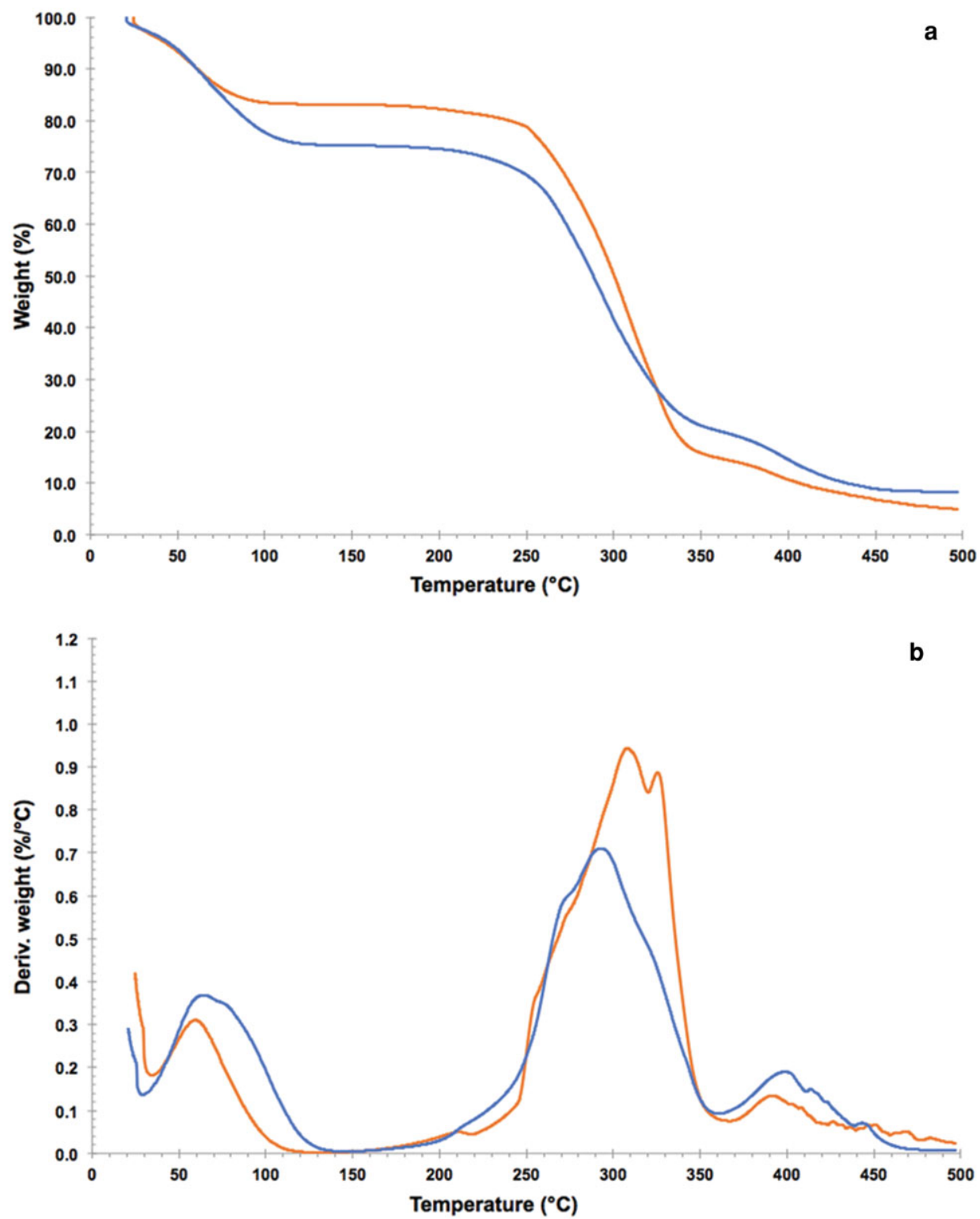

Figure 5. Thermogravimetric curves (a) and first derivative of the weight loss curves (b) of samples of hydrogel prepared according to formulation III with impregnated silver sulfadiazine (blue lines) and plain hydrogel without silver sulfadiazine (orange lines). The analyses were carried out in a thermogravimeter from TA Instruments (model 2050), using alumina pans for containing the hydrogel samples under inert (argon) atmosphere.

close agreement with those obtained from XRD studies (see Fig. 3); as can be seen from inspection of the DSC thermograms, impregnation of the hydrogel with silver sulfadiazine led to a decreased crystallinity with narrowing of the melting profile. Remarkably, the thermal events depicted in Figure 6 and the $\mathrm{X}$-ray diffractograms depicted in Figure 3 denote a clear transition from a crystalline state of the blank hydrogel (see Fig. 6, orange curve) to an amorphous counterpart (see Fig. 6, hydrogel with impregnated silver sulfadiazine, blue curve), thereby leading to the increased stability observed for the optimized antimicrobial hydrogel produced.

\section{Mathematical Modeling of Drug Release from Hydrogel}

To shed light on the release profile of silver sulfadiazine (silver sulfadiazine) from the hydrogel, and because after the 2-h timeframe of assays the amount of silver sulfadiazine released reached a plateau, one decided to apply the mathematical models of first-order $\left(\ln Q_{t}=\ln Q_{0}+k_{1} \times t\right.$, where $Q_{t}$ is the amount of drug released at time $t, Q_{0}$ is the initial amount of drug in dosage form, $k_{1}$ is a release rate constant, and $t$ is time), Higuchi $\left(Q_{t}=k_{\mathrm{H}} \sqrt{t}\right.$, where $Q_{t}$ is the amount of drug released at time $t, k_{\mathrm{H}}$ is the Higuchi constant, and $t$ is time), and KorsmeyerPeppas $\left(Q_{t} / Q_{\infty}=k_{\mathrm{KP}} t^{n}\right.$, where $Q_{t}$ is the amount of drug released at time $t, Q_{\infty}$ is the total amount of drug dissolved when the dosage form (hydrogel) is exhausted, $k_{\mathrm{KP}}$ is the KorsmeyerPeppas kinetic constant, $n$ is the diffusion or release exponent, and $t$ is time) to the drug release data, and in fact silver sulfadiazine release from the hydrogel did not occur by diffusion but by erosion of the polymeric matrix instead. This is in fact supported by the model fittings performed to the experimental 


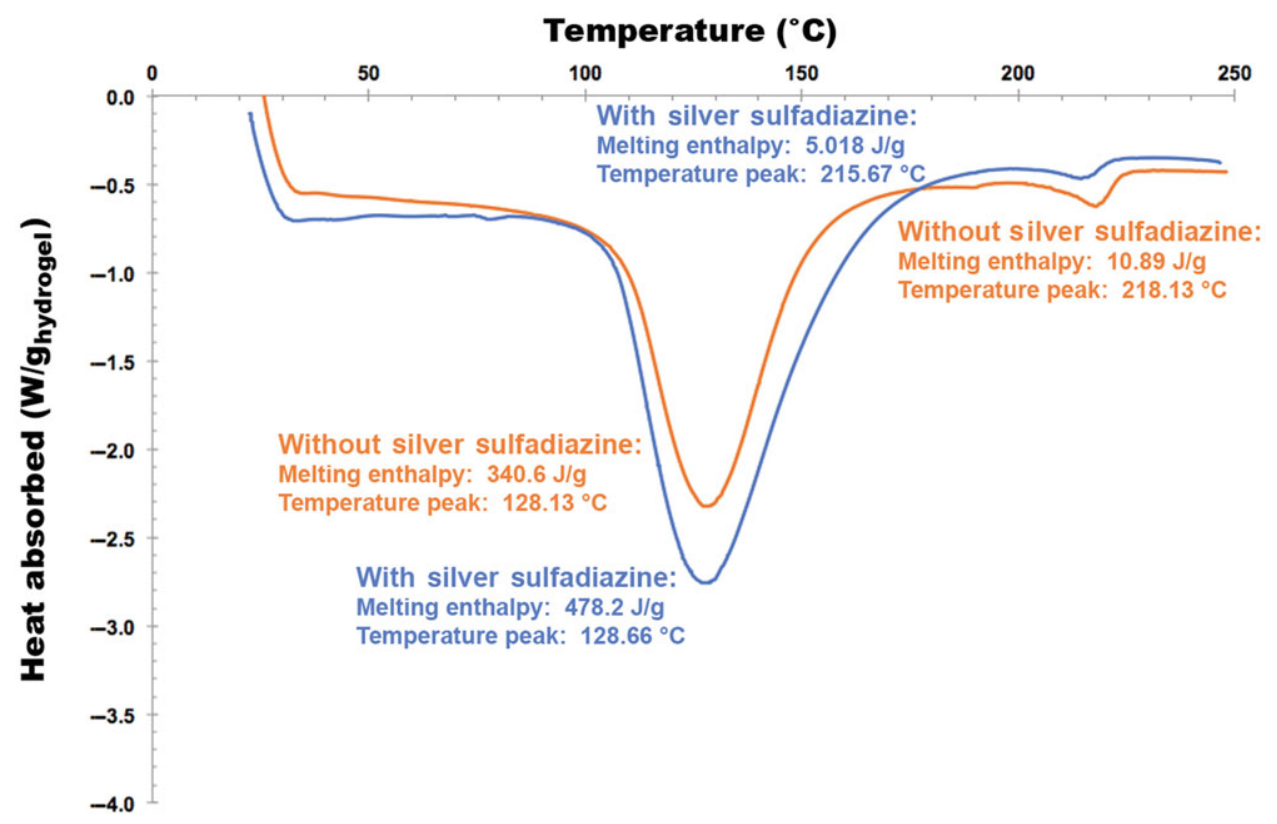

Figure 6. Differential scanning calorimetry thermograms of samples of hydrogel prepared according to formulation III with impregnated silver sulfadiazine (blue lines) and plain hydrogel without silver sulfadiazine (orange lines). The analyses were carried out in a differential scanning microcalorimeter from TA Instruments (model MDSC 2910), using high-pressure aluminum pans duly sealed by pressure.

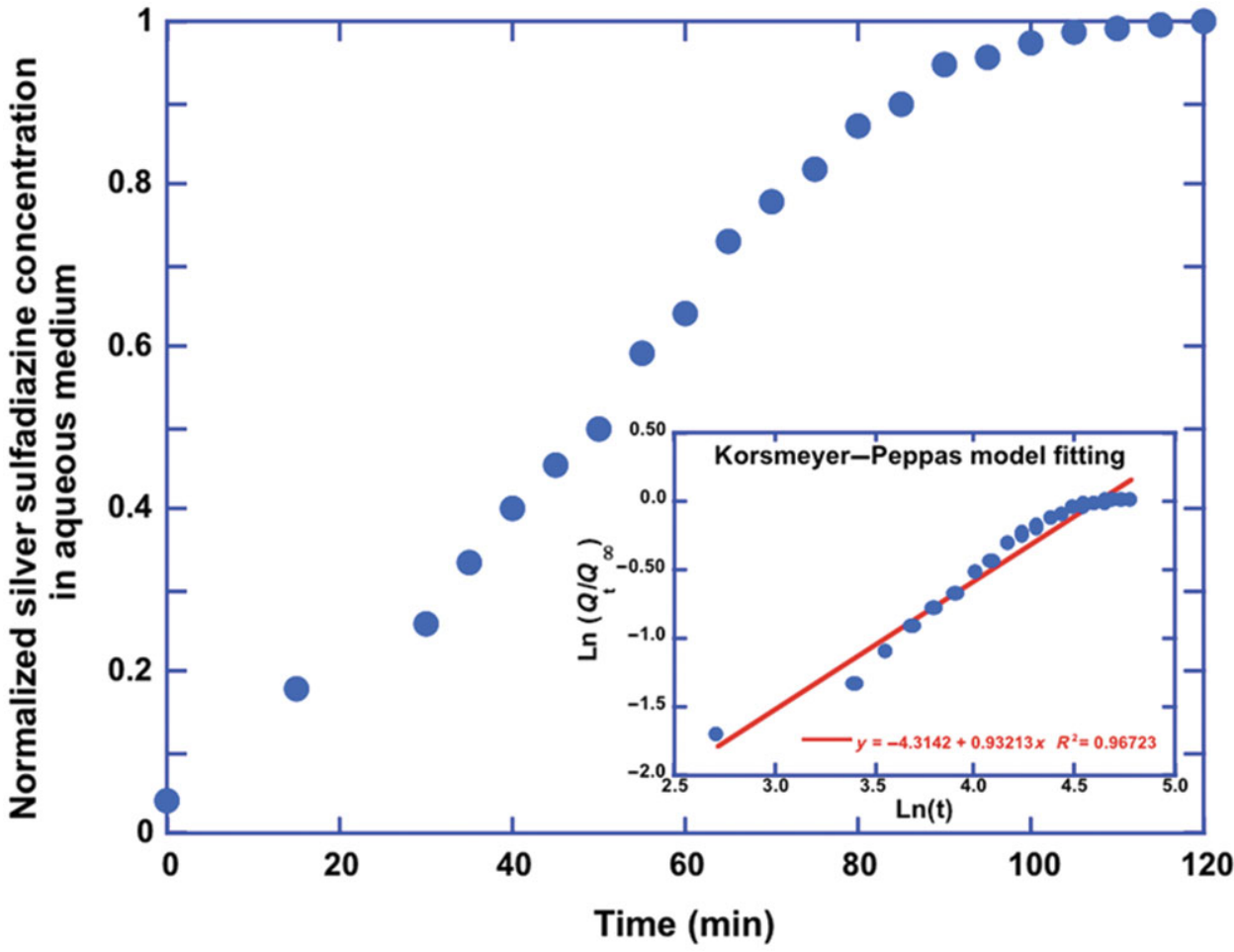

Figure 7. Normalized silver sulfadiazine concentration released from hydrogel into the aqueous medium, as function of immersion time. The inserted plot represents the linear fitting performed to transformed experimental data, using linearized Korsmeyer-Peppas model.

silver sulfadiazine release data, which produced a correlation coefficient $r^{2}=0.77257$ for the first-order model, $r^{2}=$ 0.87105 for the Higuchi model and a correlation coefficient $r^{2}$ $=0.96723$ for the Korsmeyer-Peppas model. The fittings performed clearly show a poor $r^{2}$ for both first-order and Higuchi models and an almost perfect $r^{2}$ for the Korsmeyer-Peppas model. Additionally, the diffusion or release exponent $(n)$ in the Korsmeyer-Peppas model produced by fitting the model to the silver sulfadiazine release data was $n=0.9321$ (in the Korsmeyer-Peppas drug release model, $n=0.45$ suggests Fickian diffusion, $n<0.89$ suggests anomalous diffusion or non-Fickian diffusion, and $n \geq 0.89$ suggests erosion of the 
polymeric chain), which clearly suggests that silver sulfadiazine release occurred by erosion of the hydrogel's polymeric chain. The release results (in the form of normalized silver sulfadiazine concentration in aqueous medium, see Fig. 7) have thus indicated a continuous liberation within the timeframe assayed, up to $100 \mathrm{~min}$ of immersion of the hydrogel in a lowvolume aqueous medium, time after which the silver sulfadiazine concentration maintained a plateau, thus confirming that a complete erosion of the polymeric matrix did occur. Although a slower liberation is intended for real skin wound applications, one should bear in mind that the experimental setup utilized involved a complete immersion of the hydrogel under static flow conditions. In a real situation where the hydrogel is intended for application in a skin wound, the exudation produced should not be as abundant, and thus erosion of the hydrogel with concomitant release of silver sulfadiazine should be much slower.

\section{CONCLUSIONS}

In the research effort just described, development and optimization of a hydrogel with impregnated SSDZ aiming at antimicrobial topical applications was pursued. The developed hydrogel displayed a good characteristic in relation to release of the active antimicrobial principle, verified through swelling tests, kinetics of release, and antimicrobial activity, and because release of silver sulfadiazine occurred by erosion of the hydrogel's polymeric chain, it can be utilized in occlusive wound dressings for long periods of time. The hydrogel developed can be considered as a nonadherent dressing, able to maintain a moist gel layer over the wound that is not expected to adhere, neither to the wound or the bandage, provided that it is not allowed to dry out.

\section{ACKNOWLEDGMENTS}

Project funding by Fundação de Amparo à Pesquisa do Estado de São Paulo (FAPESP, São Paulo, Brazil) (FAPESP Ref. Nos. 2012/15651-4, 2013/03181-6, and 2014/21122-0) is hereby gratefully acknowledged. This work also received support from $\mathrm{CNPq}$ in the form of a Research Productivity (PQ) fellowship granted to Victor M. Balcão. The authors have no conflicts of interest whatsoever to declare.

\section{REFERENCES}

1. Andrews GP, Laverty TP, Jones DS. 2009. Mucoadhesive polymeric platforms for controlled drug delivery. Eur J Pharm Biopharm 71(3):505-518.

2. Ganji F, Vasheghani-Farahani S, Vasheghani-Farahani E. 2010. Theoretical description of hydrogel swelling: A review. Iranian Polym J 19(5):375-398.

3. Yan C, Pochan DJ. 2010. Rheological properties of peptide-based hydrogels for biomedical and other applications. Chem Soc Rev 39(9):3528-3548.

4. Murphy DJ, Sankalia MG, Loughlin RG, Donnelly RF, Jenkins MG, McCarron PA. 2012. Physical characterisation and component release of poly(vinyl alcohol)-tetrahydroxyborate hydrogels and their applicability as potential topical drug delivery systems. Int J Pharm 423(2):326-334.
5. Song A, Rane AA, Christman KL. 2012. Antibacterial and celladhesive polypeptide and poly(ethylene glycol) hydrogel as a potential scaffold for wound healing. Acta Biomater 8(1):41-50.

6. Seabra AB, Oliveira MG. 2004. Poly(vinyl alcohol) and poly(vinyl pyrrolidone) blended films for local nitric oxide release. Biomaterials 25(17):3773-3782.

7. Sudhakar Y, Kuotsu K, Bandyopadhyay AK. 2006. Buccal bioadhesive drug delivery: A promising option for orally less efficient drugs. J Control Release 114(1):15-40.

8. Kharenko EA, Larionova NI, Demina NB. 2009. Mucoadhesive drug delivery systems. Pharm Chem J 43(4):200-208.

9. Dixit RP, Puthli SP. 2009. Oral strip technology: Overview and future potential. J Control Release 139(2):94-107.

10. Hwang M-R, Kim JO, Lee JH, Kim YI, Kim JH, Chang SW, Jin SG, Kim JA, Lyoo WS, Han SS, Ku SK, Yong CS, Choi H-G. 2010. Gentamicin-loaded wound dressing with polyvinyl alcohol/dextran hydrogel: Gel characterization and in vivo healing evaluation. AAPS PharmSciTech 11(3):1092-1103.

11. Ahmed EM. 2015. Hydrogel: Preparation, characterization, and applications. J Adv Res 6(2):105-121.

12. Liu Y, Vrana NE, Cahill PA, McGuinness GB. 2009. Physically crosslinked composite hydrogels of PVA with natural macromolecules: Structure, mechanical properties, and endothelial cell compatibility. J Biomed Mater Res B Appl Biomater 90(2):492-502.

13. Gupta P, Vermani K, Garg S. 2002. Hydrogels: From controlled release to $\mathrm{pH}$-responsive drug delivery. Drug Discov Today 7(10):569 579 .

14. İmren D, Gümüşderelioğlu M, Güner A. 2006. Synthesis and characterization of dextran hydrogels prepared with chlor- and nitrogen-containing crosslinkers. J Appl Polym Sci 102(5):42134221 .

15. Percival SL, Bowler PG, Russell D. 2005. Bacterial resistance to silver in wound care. J Hosp Infect 60(1):1-7.

16. Brasil. 2011. Ministério da saúde [Ministry oh Health]. Secretaria de Ciência e Tecnologia e Insumos Estratégicos [Secretary of Science, Technology, and Strategic Resources]. Formulário Terapêutico Nacional 2010 [National Therapeutic Form 2010]: Rename 2010. 2nd ed. Brasília: Ministério da Saúde [Ministry of Health, Brazil].

17. Lichtenstein A, Margalit R. 1995. Liposome-encapsulates silver sulfadiazine for the topical treatment of infected burns: Thermodynamics of drug encapsulation and kinetics of drug release. J Inorg Biochem 60(3):187-198.

18. Pal K, Pal S. 2006. Development of porous hydroxyapatite scaffolds. Mater Manuf Process 21(3):325-328.

19. Oliveira Junior JMR, Martins ACG. 2009. Construction and test of low cost X-ray tomography scanner for physical, chemical analysis and nondestructive inspections. AIP Conf Proc 1139:102-105.

20. Feldkamp LA, Davis LC, Kress JW. 1984. Practical cone-beam algorithm. J Opt Soc Am A 1(6):612-619.

21. Bauer AW, Kirby M, Sherris JC, Turck M. 1966. Antibiotic susceptibility testing by a standardized single disk method. Am J Clin Pathol 45:493-496.

22. Jorgensen JH, Ferraro MJ. 2009. Antimicrobial susceptibility testing: A review of general principles and contemporary practices. Clin Infect Dis 49(11):1749-1755.

23. CLSI. 2011. Performance standards for antimicrobial susceptibility testing: Twenty-first informational supplement. CLSI document M100-S21. Wayne, Pennsylvania: Clinical and Laboratory Standards Institute.

24. Clémenson S, Léonard D, Sage D, David L, Espuche E. 2008. Metal nanocomposite films prepared in situ from PVA and silver nitrate. Study of the nanostructuration process and morphology as a function of the in situ routes. J Polym Sci Part A: Polym Chem 46(6):20622071.

25. Baker MI, Walsh SP, Schwartz Z, Boyan BD. 2012. A review of polyvinyl alcohol and its uses in cartilage and orthopedic applications. J Biomed Mater Res B: Appl Biomater 100(5):14511457. 
26. Mansur HS, Sadahira CM, Souza AN, Mansur AAP. 2008. FTIR spectroscopy characterization of poly(vinyl alcohol) hydrogel with different hydrolysis degree and chemically crosslinked with glutaraldehyde. Mater Sci Eng C 28(4):539-548.

27. Yang T. 2012. Mechanical and swelling properties of hydrogels, $K T H$ Chemical Science and Engineering, Royal Institute of Technology, Stockholm, Sweden. PhD thesis, pp. 77.

28. Bourtoom T. 2008. Plasticizer effect on the properties of biodegradable blend film from rice starch-chitosan. Songklanakarin J Sci Technol 30(Suppl 1): 149-165.

29. Rippon M, White R, Davies P. 2007. Skin adhesives and their role in wound dressings. Wounds UK 3(4):76-86.

30. Pal K, Banthia AK, Majumdar DK. 2009. Polymeric hydrogels: Characterization and biomedical applications-A mini review. Des Monomers Polym 12:197-220.

31. MacGregor L. (Ed.). 2012. International consensus: Appropriate use of silver dressings in wounds. London, United Kingdom: Wounds International Enterprise House. Accessed March 19, 2015: http://www.woundsinternational.com/media/issues/567/files/content -10381.pdf.
32. Boateng JS, Matthews KH, Stevens HNE, Eccleston GM. 2008. Wound healing dressings and drug delivery systems: A review. J Pharm Sci 97(8):2892-2923.

33. Asadinezhad A, Lehocký M, Sáha P, Mozetic M. 2012. Recent progress in surface modification of polyvinyl chloride. Materials 5:2937-2959.

34. Costa Jr ES, Mansur HS. 2008. Preparação e caracterização de blendas de quitosana/poli(álcool vinílico) reticuladas quimicamente com glutaraldeído para aplicação em engenharia de tecido. Química Nova 31(6):1460-1466.

35. Skoog DA, Holler FJ, Crouch SR. 2007. Principles of instrumental analysis. 6th ed. Canada: Thomson.

36. Vila MMDC, Coelho SL, Chaud MV, Tubino M, Oliveira Jr JM, Balcão VM. 2014. Development and characterization of a hydrogel containing nitrofurazone for antimicrobial topical applications. Curr Pharm Biotechnol 15(2):182-190.

37. Vila MMDC, Tardelli ER, Chaud MV, Tubino M, Balcão VM. 2014b. Development of a buccal mucoadhesive film for fast dissolution: Mathematical rationale, production and physico-chemical characterization. Drug Deliv 21(7):530-539. 